Li Ya*, Fang Xiangqing, Liu Junyi, Dong Chi and Yang Liguo

\title{
Crystal structure of $\left(\mu_{2}\right.$-oxido)-bis $\left(N, N^{\prime}-0\right.$ - phenylenebis(salicylideneiminato))diiron(III) $-N$, $N^{\prime}$-dimethylformamide, $\mathrm{C}_{47} \mathrm{H}_{43} \mathrm{Fe}_{2} \mathrm{~N}_{4} \mathrm{O}_{9}$
}

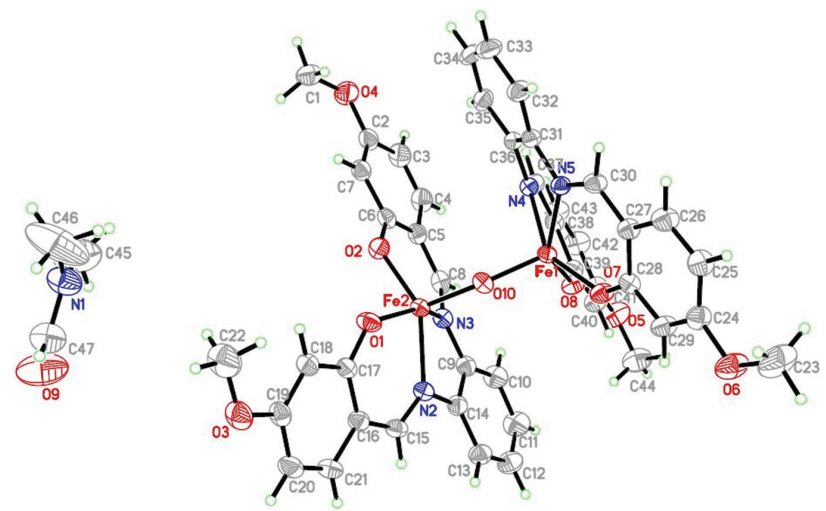

https://doi.org/10.1515/ncrs-2019-0256

Received April 8, 2019; accepted June 5, 2019; available online July 9, 2019

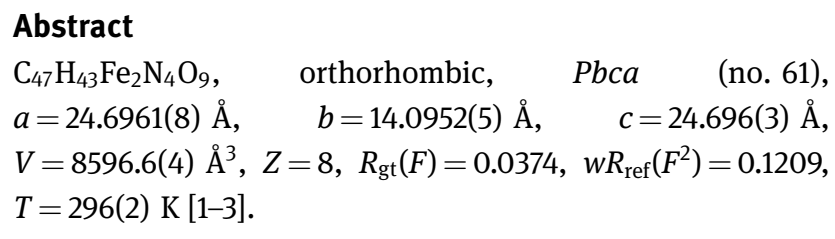

\section{CCDC no.: 1921096}

The molecular structure is shown in the figure. Table 1 contains crystallographic data and Table 2 contains the list of the atoms including atomic coordinates and displacement parameters.

\section{Source of material}

Schiff base ligand was purchased from Acros Ltd. Company and used without further purification, the other reagents were commercially available and used as purchased. The title compound was synthesized by the reaction

*Corresponding author: Li Ya, Department of Energy and Architecture, Xi'an Aeronautical University, Xi'an 710077, Shanxi, P.R. China, e-mail: 40929637@qq.com

Fang Xiangqing, Liu Junyi and Dong Chi: Department of Energy and Architecture, Xi'an Aeronautical University, Xi'an 710077, Shanxi, P.R. China

Yang Liguo: College of Chemistry and Environmental Engineering, Anyang Institute of Technology, Anyang 455000, Henan, P.R. China
Table 1: Data collection and handling.

\begin{tabular}{ll}
\hline Crystal: & Red block \\
Size: & $0.10 \times 0.08 \times 0.06 \mathrm{~mm}$ \\
Wavelength: & Mo $K \alpha$ radiation $(0.71073 \AA)$ \\
$\mu:$ & $0.74 \mathrm{~mm}^{-1}$ \\
Diffractometer, scan mode: & Bruker APEX-II, $\varphi$ and $\omega$ \\
$\theta_{\text {max }}$, completeness: & $25.0^{\circ}, 98 \%$ \\
$N(h k l)_{\text {measured }}, N(h k l)_{\text {unique }}, R_{\text {int }}:$ & $95920,7442,0.061$ \\
Criterion for $I_{\text {obs }}, N(h k l)_{\text {gt }}:$ & $I_{\text {obs }}>2 \sigma\left(I_{\text {obs }}\right), 5491$ \\
$N(\text { param })_{\text {refined }}:$ & 583 \\
Programs: & Bruker $[1]$, SHELX [2, 3] \\
\hline
\end{tabular}

Table 2: Fractional atomic coordinates and isotropic or equivalent isotropic displacement parameters $\left(\AA^{2}\right)$.

\begin{tabular}{lrrrr}
\hline Atom & $\boldsymbol{x}$ & $\boldsymbol{y}$ & $\boldsymbol{z}$ & $\boldsymbol{U}_{\text {iso }} \boldsymbol{U}_{\text {eq }}$ \\
\hline Fe1 & $0.52550(2)$ & $0.71504(3)$ & $0.63533(2)$ & $0.03486(13)$ \\
N1 & $0.79736(13)$ & $0.0119(2)$ & $0.73154(15)$ & $0.0792(9)$ \\
O1 & $0.58499(8)$ & $0.38875(14)$ & $0.63622(8)$ & $0.0514(5)$ \\
C1 & $0.53504(17)$ & $0.4742(3)$ & $0.92416(16)$ & $0.0771(11)$ \\
H1A & 0.5340 & 0.4081 & 0.9150 & $0.116^{*}$ \\
H1B & 0.5396 & 0.4810 & 0.9626 & $0.116^{*}$ \\
H1C & 0.5648 & 0.5040 & 0.9058 & $0.116^{*}$ \\
Fe2 & $0.52293(2)$ & $0.47247(3)$ & $0.64541(2)$ & $0.03739(13)$ \\
N2 & $0.49206(9)$ & $0.41138(15)$ & $0.57417(10)$ & $0.0410(6)$ \\
O2 & $0.52168(8)$ & $0.45185(14)$ & $0.72238(8)$ & $0.0475(5)$ \\
C2 & $0.47301(14)$ & $0.5166(2)$ & $0.85461(13)$ & $0.0558(9)$ \\
O3 & $0.70929(10)$ & $0.15478(16)$ & $0.57262(10)$ & $0.0695(7)$ \\
N3 & $0.43797(9)$ & $0.49383(16)$ & $0.65052(10)$ & $0.0415(6)$ \\
C3 & $0.42165(15)$ & $0.5531(2)$ & $0.84272(15)$ & $0.0642(9)$ \\
H3 & 0.3998 & 0.5774 & 0.8701 & $0.077^{*}$ \\
C4 & $0.40438(14)$ & $0.5527(2)$ & $0.79114(15)$ & $0.0585(9)$ \\
H4 & 0.3699 & 0.5756 & 0.7837 & $0.070^{*}$ \\
O4 & $0.48615(11)$ & $0.51777(18)$ & $0.90816(10)$ & $0.0739(7)$ \\
N4 & $0.50100(9)$ & $0.74156(15)$ & $0.71605(9)$ & $0.0379(5)$ \\
C5 & $0.43618(12)$ & $0.51898(19)$ & $0.74768(13)$ & $0.0453(7)$ \\
05 & $0.25958(9)$ & $0.7883(2)$ & $0.61751(11)$ & $0.0802(8)$ \\
N5 & $0.59207(9)$ & $0.78663(15)$ & $0.67104(9)$ & $0.0379(5)$ \\
O6 & $0.64832(11)$ & $0.8924(2)$ & $0.42401(9)$ & $0.0795(8)$ \\
C6 & $0.48881(12)$ & $0.48484(19)$ & $0.75972(12)$ & $0.0435(7)$ \\
07 & $0.54892(8)$ & $0.76792(14)$ & $0.56725(8)$ & $0.0482(5)$ \\
C7 & $0.50578(13)$ & $0.4821(2)$ & $0.81398(13)$ & $0.0479(7)$ \\
H7 & 0.5395 & 0.4569 & 0.8226 & $0.057^{*}$ \\
C8 & $0.41333(12)$ & $0.5175(2)$ & $0.69504(14)$ & $0.0487(8)$ \\
H8 & 0.3772 & 0.5352 & 0.6921 & $0.058^{*}$ \\
08 & $0.45020(8)$ & $0.73485(14)$ & $0.61633(8)$ & $0.0461(5)$ \\
& & & &
\end{tabular}


Table 2 (continued)

\begin{tabular}{|c|c|c|c|c|}
\hline Atom & $x$ & $y$ & $z$ & $U_{\text {iso }}{ }^{\star} / U_{\text {eq }}$ \\
\hline 09 & $0.74998(15)$ & $-0.0952(3)$ & $0.68331(15)$ & $0.1446(16)$ \\
\hline $\mathrm{C} 9$ & $0.41154(11)$ & $0.4919(2)$ & $0.60013(13)$ & $0.0457(7)$ \\
\hline $\mathrm{C} 10$ & $0.36052(13)$ & $0.5292(2)$ & $0.58843(15)$ & $0.0624(9)$ \\
\hline $\mathrm{H} 10$ & 0.3399 & 0.5568 & 0.6157 & $0.075^{\star}$ \\
\hline 010 & $0.54291(8)$ & $0.59225(12)$ & $0.63298(8)$ & $0.0441(5)$ \\
\hline $\mathrm{C} 11$ & $0.34081(15)$ & $0.5248(3)$ & $0.53633(17)$ & $0.0766(11)$ \\
\hline $\mathrm{H} 11$ & 0.3067 & 0.5495 & 0.5288 & $0.092^{*}$ \\
\hline C12 & $0.37063(15)$ & $0.4847(3)$ & $0.49538(17)$ & $0.0715(10)$ \\
\hline $\mathrm{H} 12$ & 0.3569 & 0.4834 & 0.4603 & $0.086^{*}$ \\
\hline C15 & $0.51341(11)$ & $0.3430(2)$ & $0.54649(12)$ & $0.0450(7)$ \\
\hline $\mathrm{H} 15$ & 0.4941 & 0.3216 & 0.5166 & $0.054^{\star}$ \\
\hline C14 & $0.44105(11)$ & $0.4492(2)$ & $0.55852(13)$ & $0.0456(7)$ \\
\hline C13 & $0.42053(13)$ & $0.4464(2)$ & $0.50598(14)$ & $0.0562(8)$ \\
\hline $\mathrm{H} 13$ & 0.4406 & 0.4186 & 0.4783 & $0.067^{*}$ \\
\hline $\mathrm{C} 16$ & $0.56339(12)$ & $0.29780(19)$ & $0.55735(12)$ & $0.0429(7)$ \\
\hline $\mathrm{C} 17$ & $0.59797(11)$ & $0.32341(19)$ & $0.60063(12)$ & $0.0411(7)$ \\
\hline $\mathrm{C} 18$ & $0.64806(12)$ & $0.2758(2)$ & $0.60565(13)$ & $0.0475(7)$ \\
\hline $\mathrm{H} 18$ & 0.6720 & 0.2930 & 0.6330 & $0.057^{\star}$ \\
\hline C19 & $0.66184(13)$ & $0.2040(2)$ & $0.57022(13)$ & $0.0505(8)$ \\
\hline $\mathrm{C} 20$ & $0.62657(14)$ & $0.1755(2)$ & $0.52940(13)$ & $0.0568(8)$ \\
\hline $\mathrm{H} 20$ & 0.6355 & 0.1251 & 0.5068 & $0.068^{*}$ \\
\hline $\mathrm{C} 21$ & $0.57875(13)$ & $0.2225(2)$ & $0.52302(13)$ & $0.0534(8)$ \\
\hline $\mathrm{H} 21$ & 0.5556 & 0.2044 & 0.4952 & $0.064^{\star}$ \\
\hline $\mathrm{C} 22$ & $0.74769(18)$ & $0.1793(3)$ & $0.6129(2)$ & $0.1029(16)$ \\
\hline $\mathrm{H} 22 \mathrm{~A}$ & 0.7563 & 0.2456 & 0.6100 & $0.154^{\star}$ \\
\hline $\mathrm{H} 22 \mathrm{~B}$ & 0.7800 & 0.1425 & 0.6079 & $0.154^{*}$ \\
\hline $\mathrm{H} 22 \mathrm{C}$ & 0.7328 & 0.1666 & 0.6481 & $0.154^{\star}$ \\
\hline $\mathrm{C} 23$ & $0.6877(2)$ & $0.9532(4)$ & $0.40174(17)$ & $0.1119(18)$ \\
\hline $\mathrm{H} 23 \mathrm{~A}$ & 0.6846 & 1.0149 & 0.4179 & $0.168^{\star}$ \\
\hline $\mathrm{H} 23 \mathrm{~B}$ & 0.6823 & 0.9581 & 0.3633 & $0.168^{\star}$ \\
\hline $\mathrm{H} 23 \mathrm{C}$ & 0.7231 & 0.9280 & 0.4088 & $0.168^{\star}$ \\
\hline $\mathrm{C} 24$ & $0.64485(12)$ & $0.8825(2)$ & $0.47888(13)$ & $0.0519(8)$ \\
\hline $\mathrm{C} 25$ & $0.68134(12)$ & $0.9213(2)$ & $0.51472(14)$ & $0.0554(8)$ \\
\hline $\mathrm{H} 25$ & 0.7102 & 0.9578 & 0.5025 & $0.066^{*}$ \\
\hline $\mathrm{C} 26$ & $0.67402(12)$ & $0.9048(2)$ & $0.56866(14)$ & $0.0565(8)$ \\
\hline $\mathrm{H} 26$ & 0.6986 & 0.9307 & 0.5930 & $0.068^{\star}$ \\
\hline $\mathrm{C} 27$ & $0.63106(11)$ & $0.8507(2)$ & $0.58882(12)$ & $0.0418(7)$ \\
\hline $\mathrm{C} 28$ & $0.59245(11)$ & $0.81400(19)$ & $0.55163(12)$ & $0.0400(7)$ \\
\hline $\mathrm{C} 29$ & $0.60103(12)$ & $0.8308(2)$ & $0.49690(12)$ & $0.0529(8)$ \\
\hline $\mathrm{H} 29$ & 0.5766 & 0.8067 & 0.4718 & $0.064^{\star}$ \\
\hline $\mathrm{C} 30$ & $0.62794(11)$ & $0.8370(2)$ & $0.64560(12)$ & $0.0452(7)$ \\
\hline $\mathrm{H} 30$ & 0.6541 & 0.8669 & 0.6666 & $0.054^{\star}$ \\
\hline C31 & $0.59404(11)$ & $0.77841(19)$ & $0.72797(12)$ & $0.0405(7)$ \\
\hline C32 & $0.63990(13)$ & $0.7940(2)$ & $0.75999(13)$ & $0.0530(8)$ \\
\hline $\mathrm{H} 32$ & 0.6723 & 0.8129 & 0.7441 & $0.064^{\star}$ \\
\hline $\mathrm{C} 33$ & $0.63676(15)$ & $0.7814(2)$ & $0.81518(14)$ & $0.0615(9)$ \\
\hline H33 & 0.6671 & 0.7922 & 0.8366 & $0.074^{\star}$ \\
\hline C34 & $0.58849(15)$ & $0.7524(2)$ & $0.83900(14)$ & $0.0597(9)$ \\
\hline H34 & 0.5869 & 0.7433 & 0.8763 & $0.072^{\star}$ \\
\hline C35 & $0.54300(14)$ & $0.7371(2)$ & $0.80775(13)$ & $0.0511(8)$ \\
\hline H35 & 0.5109 & 0.7174 & 0.8238 & $0.061^{*}$ \\
\hline C36 & $0.54543(12)$ & $0.75119(19)$ & $0.75225(12)$ & $0.0414(7)$ \\
\hline $\mathrm{C} 37$ & $0.45241(12)$ & $0.75649(19)$ & $0.73349(12)$ & $0.0426(7)$ \\
\hline $\mathrm{H} 37$ & 0.4481 & 0.7670 & 0.7704 & $0.051^{\star}$ \\
\hline C38 & $0.40505(11)$ & $0.75817(19)$ & $0.70095(12)$ & $0.0417(7)$ \\
\hline C39 & $0.40598(11)$ & $0.7498(2)$ & $0.64355(12)$ & $0.0408(7)$ \\
\hline
\end{tabular}

Table 2 (continued)

\begin{tabular}{lrrrr}
\hline Atom & $\boldsymbol{x}$ & $\boldsymbol{y}$ & $\boldsymbol{z}$ & $\boldsymbol{U}_{\text {iso }}{ }^{*} \boldsymbol{U}_{\text {eq }}$ \\
\hline C40 & $0.35661(11)$ & $0.7607(2)$ & $0.61482(13)$ & $0.0490(8)$ \\
H40 & 0.3566 & 0.7567 & 0.5772 & $0.059^{*}$ \\
C41 & $0.30874(12)$ & $0.7773(2)$ & $0.64180(14)$ & $0.0566(9)$ \\
C42 & $0.30764(13)$ & $0.7833(3)$ & $0.69814(15)$ & $0.0664(10)$ \\
H42 & 0.2751 & 0.7929 & 0.7162 & $0.080^{*}$ \\
C43 & $0.35528(13)$ & $0.7749(2)$ & $0.72670(14)$ & $0.0582(9)$ \\
H43 & 0.3545 & 0.7805 & 0.7642 & $0.070^{*}$ \\
C44 & $0.25620(15)$ & $0.7867(3)$ & $0.56023(16)$ & $0.0826(12)$ \\
H44A & 0.2775 & 0.8375 & 0.5455 & $0.124^{*}$ \\
H44B & 0.2191 & 0.7943 & 0.5494 & $0.124^{*}$ \\
H44C & 0.2697 & 0.7271 & 0.5470 & $0.124^{*}$ \\
C45 & $0.7528(2)$ & $0.0294(5)$ & $0.7667(2)$ & $0.146(3)$ \\
H45A & 0.7196 & 0.0146 & 0.7484 & $0.220^{*}$ \\
H45B & 0.7527 & 0.0951 & 0.7771 & $0.220^{*}$ \\
H45C & 0.7561 & -0.0095 & 0.7984 & $0.220^{*}$ \\
C46 & $0.8515(2)$ & $0.0449(5)$ & $0.7431(3)$ & $0.191(4)$ \\
H46A & 0.8769 & 0.0117 & 0.7206 & $0.287^{*}$ \\
H46B & 0.8598 & 0.0333 & 0.7805 & $0.287^{*}$ \\
H46C & 0.8537 & 0.1117 & 0.7359 & $0.287^{*}$ \\
C47 & $0.79054(18)$ & $-0.0497(3)$ & $0.69243(19)$ & $0.0873(13)$ \\
H47 & 0.8198 & -0.0594 & 0.6694 & $0.105^{*}$ \\
\hline
\end{tabular}

of $\mathrm{FeCl}_{3}$ (64.88 mg, $\left.0.4 \mathrm{mmol}\right)$ and 6,6'-((1,2-phenylenebis (azanylylidene))bis(methanylylidene))bis(3-methoxyphenol) (49.64 mg, $0.4 \mathrm{mmol})$ in DMF $(10 \mathrm{~mL})$. The mixture was stirred for $6 \mathrm{~h}$, then a colourless solution formed. The resulting solution was filtered. The filtrate was allowed to stand for a few days at room temperature until light-pink crystals were obtained. Single crystals suitable for X-ray diffraction analysis were obtained by slow evaporation of a DMF solution. (25\%, m.p. 320-325 K).

\section{Experimental details}

Hydrogen atoms were added with geometric restraints.

\section{Comment}

Organic molecules containing imine groups, commonly known as Schiff bases, are of interest to inorganic chemists as these are widely used in designing molecular ferromagnets, in catalysis, in biological modeling applications and in preparing liquid crystals [4-8]. Schiff bases also play a key role as chelating ligands in main group and transition metal coordination chemistry, due to their ease of synthesis, stability under a variety of oxidative and reductive conditions, and their structural versatility, which is associated with their applications [9-11]. Undoubtedly, salen type ligands are receiving the most attention among all the Schiff bases. Ligands categorized under this class consist of two imine nitrogen and two phenolic oxygen donors that usually coordinate in the basal plane of the metal ion, salen-type ligands are significant because of the ability of the phenoxo oxygen atoms 
to form 2-bridges, thus affording high-nuclearity compounds, which could act as promising candidates to offer valuable insight into various natural electron-transfer events [12]. Now, we synthesized the new dinuclear Fe compounds with $N, N^{\prime}$ bis(salicylidene)phenylediamine ligands. Some similar compounds have been reported [13-15].

As shown in the figure, the title compound contains a dinuclear Fe(III) complex. The compound shows a distorted octahedral geometry involving $\mathrm{N}_{2} \mathrm{O}_{2}$ donors as the basal plane. Both the Fe centers have a five coordinated square-pyramicdal geometry, in which $\mathrm{N}_{2} \mathrm{O}_{2}$ donors from the ligand and the axial position is occupied by a bridging oxygen atom.

Acknowledgements: The authors gratefully acknowledge the financial support by Young Talent fund of University Association for Science and Technology in Shaanxi, China, Educational Commission of Shaanxi Province of China (16 J K1393) and Science Foundation of Shaanxi Province of China (2016JQ2001).

\section{References}

1. Bruker. SMART and SAINT for Windows NT Software Reference Manuals, Version 5.0. Bruker Analytical X-Ray Systems, Madison, WI (1997).

2. Sheldrick, G. M.: SADABS, a software for empirical absorption correction. University of Göttingen, Göttingen, Germany (1997).

3. SHELXL. Reference Manual, version 5.1; Bruker Analytical X-Ray Systems, Madison, WI (1997).

4. de Barbarin, C. O. R.; Bailey, N. A.; Fenton, D. E.; He, Q. Y.: Zinc (II) complexes derived from potentially hexadentate $\left(\mathrm{N}_{4} \mathrm{O}_{2}\right)$ acyclic ligands containing pyridinyl andphenolic groups. J. Chem. Soc. Dalton Trans. 2 (1997) 161-166.

5. Larson, E. J.; Pecoraro, V. L.: The peroxide-dependent $\mu_{2}-0$ bond formation of manganese complex $[\mathrm{Mn}(\mathrm{IV}) \operatorname{SALPN}(0)]_{2}$. J. Am. Chem. Soc. 113 (1991) 3810-3818.
6. Khan, M. M. T.; Srinivas, D.; Kureshy, R. I.; Khan, N. H.: Synthesis, characterization, and EPR studies of stable ruthenium (III) Schiff base chloro and carbonyl complexes. Inorg. Chem. 29 (1990) 2320-2326.

7. Miyasaka, H.; leda, H.; Mastumoto, N.; Crescenzi, R.; Floriani, C.: Assembling bi-, tri-and pentanuclear complexes into extended structures using a desolvation reaction: synthesis, structure, and magnetic properties of manganese(III) Schiff-Base hexacyanoferrate polymeric compounds and their derived extended structures. Inorg. Chem. 37 (1998) 255-263.

8. Ramade, I.; Kahn, O.; Jeannin, Y.; Robert, F.: Design and magnetic properties of a magnetically isolated GdIIICull pair. crystal structures of [Gd(hfa) ${ }_{3} \mathrm{Cu}($ salen) $],\left[\mathrm{Y}(\mathrm{hfa})_{3} \mathrm{Cu}(\right.$ salen)], [Gd(hfa) ${ }_{3} \mathrm{Cu}\left(\right.$ salen)(Meim)], and [ $\mathrm{La}(\mathrm{hfa})_{3}(\mathrm{H} 2 \mathrm{O}) \mathrm{Cu}($ salen)] [hfa $=$ Hexafluoroacetylacetonato, salen $=N, N^{\prime}$-ethylenebis (salicylideneaminato), Meim =1-Methylimidazole]. Inorg. Chem. 36 (1997) 930-936.

9. Ziessel, R.: Schiff-based bipyridine ligands. unusual coordination features and mesomorphic behaviour. Coord. Chem. Rev. 216 (2001) 195-223.

10. Cozzi, P. G.: Metal-salen Schiff base complexes in catalysis: practical aspects. Chem. Soc. Rev. 64 (2004) 410-421.

11. Gupta, K. C.; Sutar, A. K.: Catalytic activities of Schiff base transition metal complexes. Coord. Chem. Rev. 252 (2008) 1420-1450.

12. Katsuki, T.: Catalytic asymmetric oxidations using optically active (salen)manganese(III) complexes as catalysts. Coord. Chem. Rev. 140 (1995) 189-214.

13. Jana, S.; Chatterjee, S.; Chattopadhyay, S.: Syntheses, characterization and X-ray crystal structures of hexa-coordinated monomeric and oxo-bridged dimeric Fe(III) compounds with salen-type Schiff bases. Polyhedron 48 (2012) 189-198.

14. Meng, Q.; Clegg, J. K.; Brock, A. J.; Jolliffe, K. A.; Lindoy, L. F.; Wei, G.: Mono-and dinucleating $\mathrm{Ni}(\mathrm{II}), \mathrm{Cu}(\mathrm{II}), \mathrm{Zn}(\mathrm{II})$ and $\mathrm{Fe}(\mathrm{III})$ complexes of symmetric and unsymmetric Schiff bases incorporating salicylimine functions-synthetic and structural studies. Polyhedron 74 (2014) 113-121.

15. Oyaizu, K.; Dewi, E. L.; Tsuchida, E. A.: $\mu$-Oxo diiron(III) complex with a short Fe-Fe distance: crystal structure of ( $\mu$-oxo) bis $\left[N, N^{\prime}\right.$-o-phenylenebis(salicylideneiminato)iron(III)]. Inorg. Chim. Acta 321 (2001) 205-208. 